\title{
The Initial Case of COVID-19 - Shulan City, Jilin Province, China, May 8, 2020
}

\author{
Cao Chen ${ }^{1,2, x}$; Xiang Zhao ${ }^{1,2, x}$; Dayan Wang'; Juan $\mathrm{Li}^{4} ;$ Ao Wang'; Donglin Wu ${ }^{3}$; Zhixiao Chen'; Yuchao Wu'; Yao \\ Meng'; Weifeng Shi'; George F. Gao ${ }^{1,2}$; Guizhen $\mathrm{Wu}^{1,2, * ;}$; Biao Huang ${ }^{3, * ;}$; Wenbo $\mathrm{Xu}^{1,2, *}$
}

Coronavirus disease 2019 (COVID-19) was designated a global pandemic by the World Health Organization (WHO) on March 11, 2020 (1). After great effort, COVID-19 has been well-controlled in China, but new challenges have emerged due to increasing numbers of imported cases from outside of China. On May 8, the Health Commission of Jilin Province reported a confirmed COVID-19 case of a 45-year-old laundry woman from Shulan City in the northeast of China. This case was suspected to be associated with a possible importation event.

On May 10, genome sequencing was performed at China CDC using the Illumina MiSeq platform. Due to the low viral load in the samples, $98.1 \%$ of the virus genome sequence was obtained after sequencing and assembly, which included the key sites of the virus, and the analysis of the potential source of the virus was not affected. Compared with the reference sequence EPI_ISL_402119 (2), which was isolated from Wuhan on January 7, 2020, the virus had mutations at C241T, C3037T, C14408T, and A23403G, and the GGG mutated to AAC at position 28881-28883 of nucleotide of COVID-19 virus, which shows gene mutation characteristics of the L-lineage from Europe and does not have characteristic mutations of the North America branch of the European L-lineage (mutants at C1059T and G25563T) according to the study of Tang et al (Figure 1) (3). Additionally, the genome sequence of such a case also had a mutation at T19839C.

The result of viral genome sequencing provides strong evidence that the first COVID-19 case in Shulan City is related to COVID-19 virus imported from Europe. Although isolation of the imported cases and close contacts are the top priority strategies to interrupt the spread of the virus in China, asymptomatic infected persons may be a potential source of infection for this case or an unknown source of sporadic cases. We suggest that COVID-19 virus nucleic acid testing for respiratory and stool specimens of the imported cases, close contacts, and inbound passengers whom come from high risk countries, territories, and areas should continue to be strengthened. Only when the nucleic acid tests of respiratory and stool specimens are negative at the same time, the isolation can be ended. Epidemiological investigation, case treatment of asymptomatic infections, and close contact tracing management should be continuously accelerated.

Fundings: This work was supported by National Key Research and Development Program of China (Program No. 2018YFC1200305), National Science and Technology Major Project of China (Project No. 2018ZX10102001, 2018ZX10711001, 2018ZX1071 3002).

doi: $10.46234 / \mathrm{ccdcw} 2020.115$

\# Corresponding authors: Guizhen Wu,wugz@ivdc.chinacdc.cn; Biao Huang, huangbiao2000@sohu.com; Wenbo Xu, xuwb@ivdc. chinacdc.cn.

\footnotetext{
${ }^{1}$ National Health Commission Key Laboratory for Medical Virology and Viral Diseases, National Institute for Viral Disease Control and Prevention, Chinese Center for Disease Control and Prevention, Beijing, China; ${ }^{2}$ Center for Biosafety Mega-Science, Chinese Academy of Sciences, Wuhan, China; ${ }^{3}$ Jilin Provincial Center for Disease Control and Prevention, Jilin, China; ${ }^{4}$ Key Laboratory of Etiology and Epidemiology of Emerging Infectious Diseases in Universities of Shandong, Shandong First Medical University, and Shandong Academy of Medical Sciences, Taian, China.

\& Joint first authors.
}

Submitted: June 02, 2020; Accepted: June 03, 2020

\section{REFERENCES}

1. World Health Organization. Coronavirus disease (COVID-19) situation report-133. https://www.who.int/docs/default-source/coronaviruse/situa tion-reports/20200601-covid-19-sitrep-133.pdf. [2020-06-01].

2. Zhu N, Zhang DY, Wang WL, Li XW, Yang B, Song JD, et al. A novel coronavirus from patients with pneumonia in China, 2019. New England J of Med. 2020; 382(8):727 - 733.

3. Tang XL, Wu CC, Li X, Song YH, Yao XM, Wu XK, et al. On the origin and continuing evolution of SARS-CoV-2. Nat Sci Rev 2020 http://dx.doi.org/10.1093/nsr/nwaa036. 


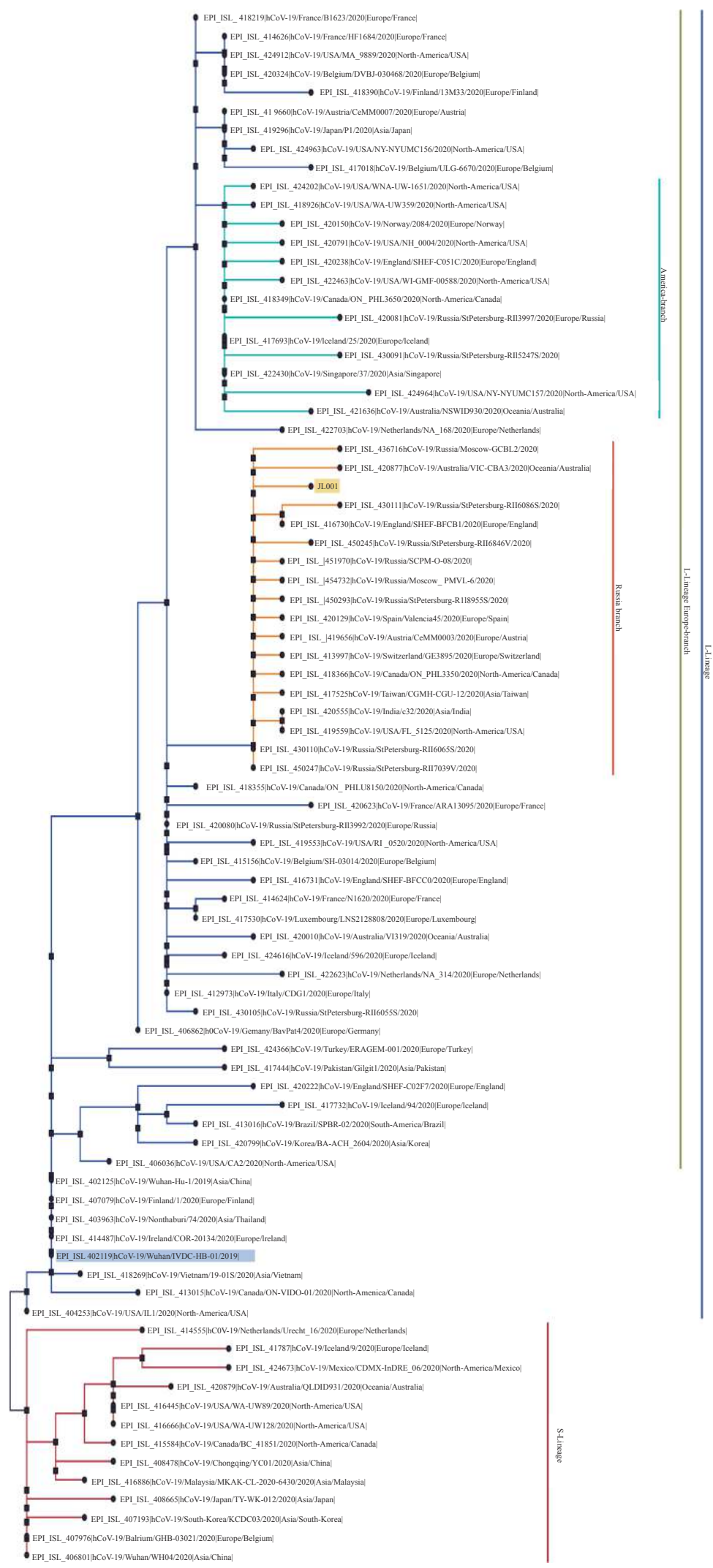

FIGURE 1. Phylogenetic tree based on the genome sequences of representative COVID-19 viruses. The genome of COVID-19 virus of the initial case in Shulan City and those from Wuhan City were highlighted with the shade of yellow and blue, respectively. S- or L-lineage of COVID-19 virus were marked and colored on the right. 